\title{
A ferrofluid inclinometer with a time domain readout strategy
}

\author{
B. Andò, S. Baglio, A. Beninato \\ DIEEI, University of Catania, Catania, Italy
}

\begin{abstract}
In this paper a tilt sensor exploiting the effect of the imposed tilt on a ferrofluid drop free to move inside a glass channel is presented. The proposed inclinometer consists of a $10 \mathrm{~mm}$ diameter pipe containing a $0.25 \mathrm{ml}$ of ferrofluid, an actuation coil to control the ferrofluid mass movement and two sensing coils to monitor the mass position. The actuation and the sensing coils are wound around the pipe and the length of the actuation/sensing system is $78 \mathrm{~mm}$. The novelty of this device, as respect to solutions already presented in the literature, consists in the conversion of the device tilt into the time elapsed between transitions of the ferrofluid mass in two fixed positions inside the channel. This time domain readout strategy allows to implement a digital form of the device output which can be intended as the main advantage of the solution proposed.
\end{abstract}

C 2012 The Authors. Published by Elsevier Ltd. Selection and/or peer-review under responsibility of the Symposium Cracoviense Sp. z.o.o. Open access under CC BY-NC-ND license.

Keywords: flow sensor; ferrofluid active mass; inductive readout;

\section{Introduction}

Ferrofluids (also called magnetic fluids or magnetic nanofluids) are a special category of nanomaterials which exhibit simultaneously liquid and superparamagnetic properties. Due to the fact that no natural liquids offer these features, the starting point of the field of magnetic fluid research can be found in a work of Resler and Rosensweig [1]. Such a work shows that a liquid material with controllable magnetic properties can be used in many applications. Successively, strong efforts have been undertaken to synthesize a system enabling the mentioned magnetic control. Modern ferrofluids are colloidal magnetic fluids. They are composed by small particles of solids, magnetic, single-domain particles coated with a molecular layer and a dispersant (surfactant), and suspended in a liquid carrier. The particles are sufficiently small so that the ferrofluid retains its liquid characteristics even in the presence of a magnetic field, and substantial magnetic forces can be induced which results in fluid motion. Interesting examples of ferrofluid transducers are presented in literature [2-3]. 
In this paper a novel approach to implement a tilt sensor is presented. The proposed approach is based on a ferrofluid mass continuously moving back and forth inside a glass channel, while the sensing methodology exploits the influence of the applied tilt on the transition time of the ferrofluid mass between two fixed sites.

The novelty of this device as respect to solutions already available in the literature is the exploitation of a Time Domain Readout (TDR) strategy, implemented through a low cost sensing approach, which provides the system with an intrinsic digital form of the output signal.

Actually, the tilt information is achievable through the observation of the time elapsed (the transition time) by the ferrofluid mass in the transition between two fixed sections of the pipette. An inductive readout strategy and a conditioning electronics driven by a carrier signal are used to sense the position of the ferrofluid mass inside the channel. The system output signal is the driving carrier signal modulated by the ferrofluid position. The transition time is evincible by observing the frequency of the demodulated output signal.

A main outcome of the proposed device is the possibility to exploit its output in the frequency domain to simply operate with digital elaboration systems, such as microcontroller based architecture.

Other advantages of the proposed inclinometer are related to the use of ferrofluid as the inertial mass of the system which confers high robustness against mechanical shocks and the possibility to implement the sensing strategy also in pre-existing channels, by injecting a mass of ferrofluid and clamping the external control and the sensing units.

The electric isolation between the device electronics (sensing and actuation) and the liquid media and the disposable characteristic of the device, given by the physical decoupling between the cheap device structure (pipe and ferrofluid) and the re-usable sensing/actuation system, allow the use of the proposed sensor in wide real scenario.

Among other application contexts, the device can be conveniently adopted in the biomedical field.

\section{The device}

The TDR (Time Domain Readout) inclinometer consists of a glass pipe containing a ferrofluid mass dispersed in water, two sensing coils to monitor the mass position and an actuation coil whose task is twofold: it controls the ferrofluid mass and acts as the excitation coil of the inductive sensing architecture. Both the actuation and the sensing coils are wound around the pipe, as schematized in Fig. 1: the actuation coil is positioned in the centre of the pipe while the sensing coils are symmetrically just opposed as respect to the actuation coil and connected in a differential configuration. The basic idea is to exploit the relationship between the imposed tilt and the time elapsed during the transition of the ferrofluid mass between two fixed positions shown in Fig. 1: the centre of the actuation coil (P1) and a fixed position inside one of the two sensing coils $\left(\mathrm{P} 2_{\mathrm{L}, \mathrm{R}}\right)$. The actuation coil is driven by an excitation current

$$
i_{\text {exc }}=I_{0}+I_{M} \sin (\omega t)
$$

The $I_{0}$ current is used to implement a retaining mechanism which assures a sucking effect on the ferrofluid mass towards the centre of the actuation coil. When the DC current $I_{0}$ is turned on, the ferrofluid mass experiences a magnetic force able to suck the ferrofluid volume towards the centre of the coil independently on its current position inside the pipe. The sinusoidal term represents the carrier signal used to excite the inductive readout architecture (sensing coils), while its effect on the retaining mechanism is negligible. 
In the case of a null tilt the mass resides in its equilibrium position in the centre of the actuation coil. When a tilt is imposed to the device and $I_{0}=0$ the mass moves towards the end of the pipe. When the mass reaches one of the two $\mathrm{P} 2_{\mathrm{L}, \mathrm{R}}$ control positions, the $I_{0}$ restoring current is turned on in order to produce a magnetic force sucking the mass towards the $\mathrm{P} 1$ position. The presence of the ferrofluid mass in the $\mathrm{P} 2_{\mathrm{L}, \mathrm{R}}$ control position is detected by the sensing coils and the $I_{0}$ restoring current is activated by comparing the output signal of the readout electronics with fixed thresholds.

When the mass reaches the $\mathrm{P} 1$ position, $I_{0}$ is switched off and the mass moves again along the channel towards the $\mathrm{P} 2 \mathrm{~L}, \mathrm{R}$ control positions.

The Transition Time elapsed by the ferrofluid mass to move from the centre of the actuation coil to the control position depends on the tilt magnitude.

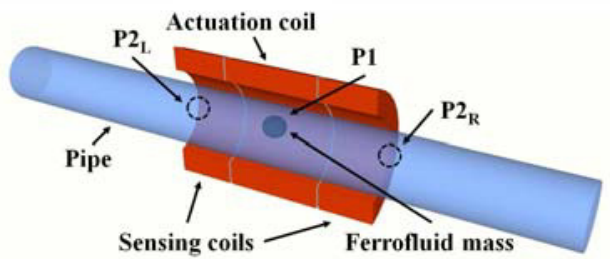

Fig. 1. Schematization of the developed inclinometer.

It must be observed that the continuous movement of the ferrofluid mass between the centre of the actuation coil and the control positions prevents potential static friction and the possible adhesion of the ferrofluid mass into the channel walls.

The output voltage of the readout electronics is given by the signal driving the sensing coils modulated by the movement of the ferrofluid mass between the $\mathrm{P} 1$ and $\mathrm{P} 2_{\mathrm{L}, \mathrm{R}}$ positions.

As sketched in Fig. 2, information on the mass movement is achievable by demodulating the output signal of the conditioning electronics and, as above mentioned, the frequency of the demodulated signal is strictly related to the device tilt.

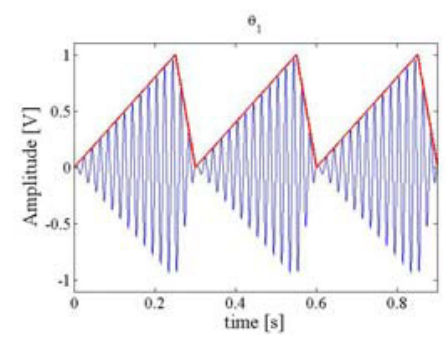

(a)

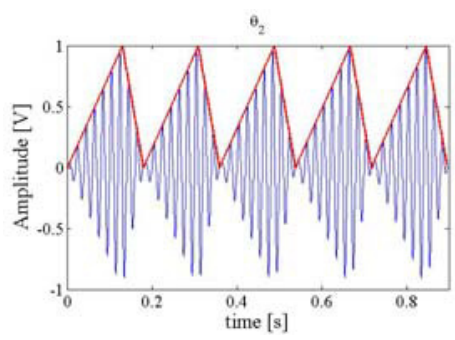

(b)

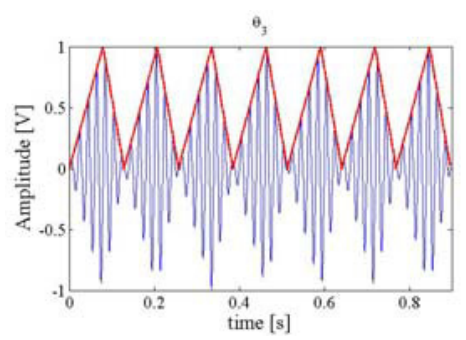

(c)

Fig. 2. Trends expected of the device output signals for different tilt values $(\theta 1<\theta 2<\theta 3)$. Information on the ferrofluid mass movement is contained in the modulating signal.

\section{Experimental results}

A real view of the sensor prototype is shown in Fig. 3a where the pipe, the two sensing coils and the actuation coil are evincible.

The ferrofluid mass transitions in $\mathrm{P} 2_{\mathrm{L}, \mathrm{R}}$ are detected by the sensing coils which are driven by a $200 \mathrm{mV}$ signal@3 kHz through a AC bridge configuration. 
In order to impose the $I_{0}$ bias value an arbitrary waveform generator controlled by a computer through the IEEE 488 standard is used, that enables or disables the offset value, while the output signal is acquired through a DAQ acquisition board. A scheme of such a working principle is shown in Fig. $3 \mathrm{~b}$.

The acquired signal is given by the carrier driving the readout electronics modulated by the ferrofluid mass movement. The information related to the mass displacement is obtained by demodulating the acquired signal and the frequency of the demodulated signal is strictly related to the applied tilt.

In order to implement the experimental set-up for the device characterization a step motor with a minimum step size of $0.06^{\circ}$ was used as well as a reference system to independently estimate the imposed tilt. A set of experimental observations was performed by tilting the device in a range between $0^{\circ}$ and $13.20^{\circ}$.

The calibration diagram of the inclinometer prototype showing the relationship between the imposed tilt, $\theta$, and the frequency, $v$, of the demodulated signal is given in Fig. 4. Sensitivity and resolution estimated by the experimental characterization are $0.08 \mathrm{~Hz} /{ }^{\circ}$ and $0.25^{\circ}$, respectively.

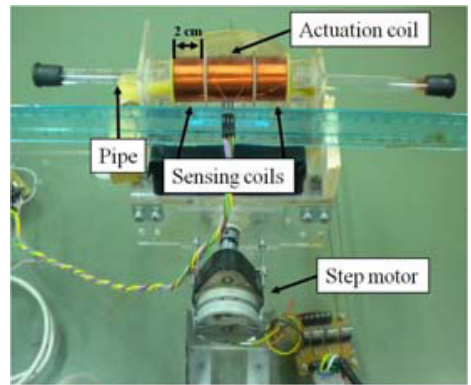

(a)

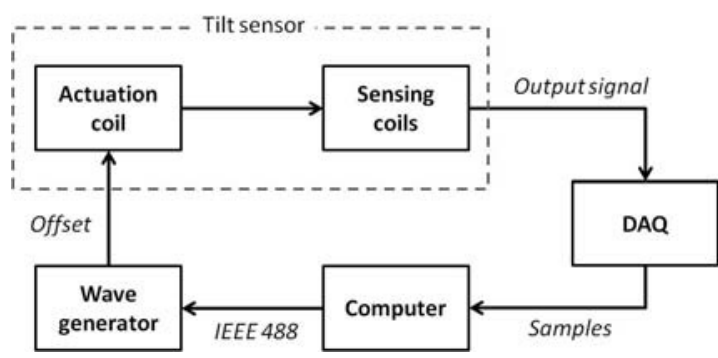

(b)

Fig. 3. (a) Real view of the developed prototype and (b) scheme of the working principle of the developed system.

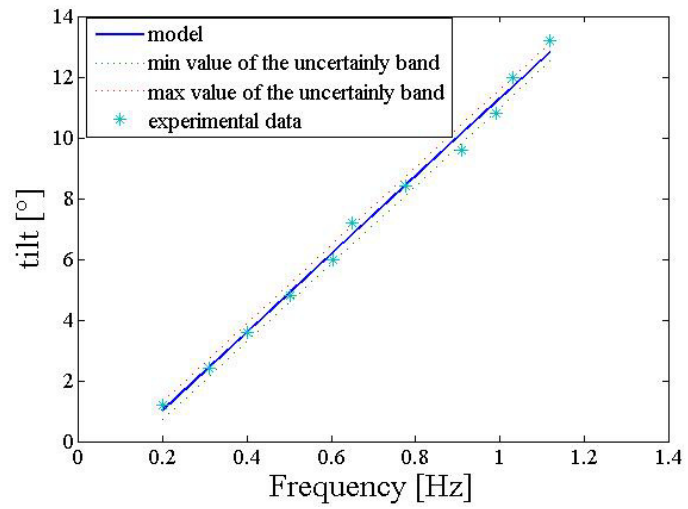

Fig. 4. Calibration diagram of the inclinometer.

\section{References}

[1] E. L. Jr. Resler and R. E. Rosensweig, "Magnetocaloric power". AIAA J., 2, 8 pp. 1418, 1964.

[2] B. Andò, A. Ascia, S. Baglio, N. Savalli, "A Novel Ferrofluidic Inclinometer", IEEE Transaction on Instrumentation and Measurement, Vol. 56, No. 4,pp. 1114-1123, August 2007.

[3] B. Andò, S. Baglio, A. Beninato, Behavior Analysis of Ferrofluidic Gyroscope Performances, Sensors \& Actuators: A. Physical Vol. 162, Issue 2, pp. 348-354, 2010. 